\title{
Theoretical and experimental analysis on the thermal fluid dynamics of water droplets in irrigation
}

\author{
G. Lorenzini ${ }^{1} \&$ D. De Wrachien ${ }^{2}$ \\ ${ }^{I}$ Department of Agricultural Economics and Engineering, \\ Alma Mater Studiorum-University of Bologna, Italy \\ ${ }^{2}$ Department of Agricultural Hydraulics, University of Milan, Italy
}

\begin{abstract}
A complete thermal fluid dynamics analysis of a sprinkler droplet following its path from the sprinkler nozzle to the ground is made difficult by the high nonlinearity of the differential equations describing the phenomenon. This fact, caused by a great inter-dependence between the parameters that play a role in the process, is partially overcome in this paper by representing the process in terms of force balance to which a few simplifying hypotheses are applied. The goal of this approach is to make the description entirely analytical thus avoiding any empiricisms that could limit the generality of the study. The model realised is able to provide reliable kinematic data, which prove to match significantly with data available in literature, especially for higher Reynolds numbers. The paper also shows an application of the model to the computation of the aerial evaporation of a water droplet: quantitatively, this part of the study is able to provide an upper limit of the friction-induced phenomenon only, however qualitatively the consequent analysis of the results opens a new window on the full understanding of the aerial evaporation of sprinkler water, highlighting the possible role played by certain environmental parameters, such as air friction and air temperature. This latter analysis also involved careful experimental activity, which is also presented herein.
\end{abstract}

Keywords: thermal fluid dynamics, mathematical model, sprinkler irrigation, water droplet, travel distance, time of flight, evaporation. 


\section{Introduction}

It is a widely reported fact that in industrialised countries more than half of the freshwater available is used for agricultural purposes and for crop irrigation in particular. This implies that the important challenge of achieving a more sustainable management of water, called for by the increasingly worrying overexploitation of this resource, necessarily entails more efficient agricultural practices especially with regard to irrigation in general and sprinkler irrigation in particular, which is the key issue of this paper. From a technical physics point of view, the need to save water in sprinkler irrigation requires efforts in understanding and fully describing the whole phenomenon of a water droplet exiting a sprinkler nozzle, following its path and finally reaching the soil.

The general problem, characterised by the many interacting factors in determining the trajectory and evaporation of an airborne water droplet, can be summarised as follows:

- Experimentally [1-3], it is difficult to compute the effect of each single environmental parameter on aerial droplet dynamics, by distinguishing its effect from those of the other affecting parameters. In particular, when experimentally investigating aerial evaporation of a droplet, the results are often expressed by small numbers or percentages, the reliability of which depend on the typical error of measurement limits, which are sometimes of the same order of magnitude as the computed values.

- Analytically [4-6], a mutual interaction of the affecting parameters means that a complete description of the phenomenon and of the interdependences between parameters requires a non-linear partial differential equation to be solved: it is very unlikely that this will lead to a final solution, unless the procedure resorts significantly to casedependent empiricisms, however, this option adds very little information to the general comprehension of the phenomenon.

The potential solutions to these problems, which are examined in this paper, imply:

- Experimentally, it would be necessary to perform single-parametric research on the process, that is evaluating the effect on spray dynamics of each affecting parameter independently of all the others and thus minimising the effect of all variables except that investigated. This is the approach adopted in this paper, for the affect of air temperature, with meticulous test management and data collection.

- Analytically, great efforts would be required to simplify the modelling of sprinkler water droplet dynamics, realising models of general (that is entirely analytical) and easy (that is considering a limited range of variables) applicability, but that also provide a reliable description of the actual phenomena: to this ends, in our research, we applied a mathematical model, based on a simplified force balance, that describes the aerial path of the process examined providing results that match satisfactorily with other authors' data. 
The main goals of this paper are:

- Experimentally, to show a method, applied in this case to the analysis of the effect of air temperature, which, when suitably expanded, could be a potent technique for determining each single parametrical contribution to the global phenomenon.

- Analytically, to provide a fully analytical (in the hypotheses formulated) tool that can describe in-field events with a good degree of match to actual data and which is significantly easier (in the sense explained above) than the approaches available in literature and quoted in this work.

The results obtained seem to encourage an attempt to re-write the physics of the whole process under examination, also regarding the computation of the aerial evaporation, phenomenon for which some computed and experimental results (in the sense explained below) are also presented.

\section{Materials, methods and results}

\subsection{Kynematics}

A new simplified approach to the kinematic modelling of water droplet flow in sprinkler irrigation is that provided by Lorenzini [7], who describes the flow of a single sprinkler droplet based on the force balance: $\vec{F}=m \vec{a}$, where $\vec{F}$ is the total force acting on the droplet and equal to the vectorial sum of the weight of the droplet of mass $m$ diminished by its buoyancy force and of the friction force acting during the flight on the droplet of acceleration $\vec{a}$. The friction factor $f$ used in the model is that according to Fanning's definition [8]. The hypotheses formulated are that:

- Each droplet is generated exactly at the nozzle outlet

- The forces applied to the system are weight, buoyancy and friction

- The droplet has a spherical shape for the whole trajectory

- The volume of the droplet does not vary during the flight

- Friction has the same direction as droplet velocity but opposite sense for the whole path

- There is no wind disturbing the flight.

The parameters, depending on the practical case considered, to be introduced for the computation of the results are:

- The nozzle height $h$ from ground level

- The droplet exit (from the nozzle) velocity $v_{0}$ and the angle $\alpha$, in relation to the horizontal direction, at which the jet is initially inclined.

If $n$ is the weight of the droplet accounting for its buoyancy component, $k=\frac{f \rho A}{2}$ (where $\rho$ is air density, which is dependent on temperature, and $A$ is the cross section of the droplet) is the coefficient that defines the action of the friction force and $g$ is the acceleration of gravity, then the balance in final form is: 


$$
\left(-k \dot{x}^{2},-k \dot{y}^{2}-n g\right)=m(\ddot{x}, \ddot{y})
$$

which, in the horizontal and vertical directions, respectively, gives:

$$
\begin{gathered}
m \ddot{x}=-k \dot{x}^{2} \\
\ddot{y}=-k \dot{y}^{2}-n g
\end{gathered}
$$

where $\dot{x}, \dot{y}, \ddot{x}, \ddot{y}$ are velocities and accelerations in the horizontal and vertical direction, respectively. The initial conditions defined are $x(t=0)=0$ and $\dot{x}(t=0)=v_{0 x}$ for the first equation, whereas $y(t=0)=h$ and $\dot{y}(t=0)=v_{0 y}$ for the second. Where: $t$ is time; $v_{0 x}, v_{0 y}$ are the horizontal and vertical velocity components, respectively, at the exit of the nozzle. Integrating the system of differential equations gives the full analytical solution of the problem in the form of parametric equations of position $(x(t), y(t))$, velocity $(\dot{x}(t), \dot{y}(t))$ and time of flight $\tau$. This model, by providing an exact solution, applies to many cases but in the hypotheses formulated only. It should be pointed out that the parameter $k$ has to be managed carefully according to the flow state considered: in fact it may be that a droplet starts its path in a certain flow state, modifying it along the way, thus requiring a different form of $k$ (as explained above) to be introduced into the model. The validation of the model proposed needs a quantitative approach to determine how reliable the predictions are: this can be achieved by introducing other authors' data into the model. The research work chosen for comparative purposes is that of Edling [5] and Thompson et al. [3]: among the cases studied by these authors, only those involving a no-wind condition were considered. Results are shown in Figs. 1 to 10 in terms of travel distance and time of flight. In Figs. 4 to 9, it clearly a shows very good agreement in most cases. This does not hold true in Figs. 1, 2 and 3 for a droplet diameter of $0.5 \times 10^{-3} \mathrm{~m}$ : in these cases, in any case, Edling's [5] data could not be entirely reliable being numerically too close together regardless of parameter variations. Figures 10 and table 1 show the comparative analysis on the basis of Thompson et al.'s [3] data in terms of travel distance and time of flight, respectively. A difference can be noted for the results for droplet diameter of $0.3 \times 10^{-3} \mathrm{~m}$ : this is related to the flow description adopted in [3] for smaller droplets, which was not shared in the present approach. The other data, particularly those referring to intermediate droplet diameters in the range, show reasonable agreement both in the values obtained and in the trends determined. These comparisons show that the model defined here proves to be kinematically reliable in its predictions even from a quantitative point of view. This result is particularly relevant as its construction excluded most of the complicated parameters typically introduced in other models to describe the same phenomenon and to obtain similar results. 


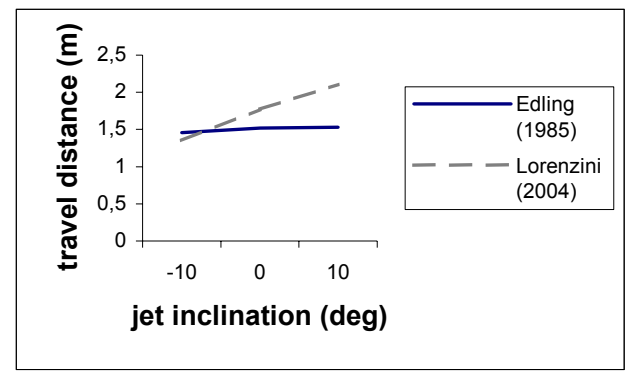

Figure 1: Travel distance of sprinkler droplets: Edling's [5] data compared to Lorenzini's [7]: flow rate $=1.4 \cdot 10^{-4} \mathrm{~m}^{3} \cdot \mathrm{s}^{-1}$; nozzle diameter $3.96 \cdot 10^{-3} \mathrm{~m}$; air temperature $29.4^{\circ} \mathrm{C}$; nozzle height $=1.22 \mathrm{~m}$; droplet diameter $=$ $0.5 \cdot 10^{-3} \mathrm{~m} .\left(\mathrm{R}^{2}=0.946\right)$.

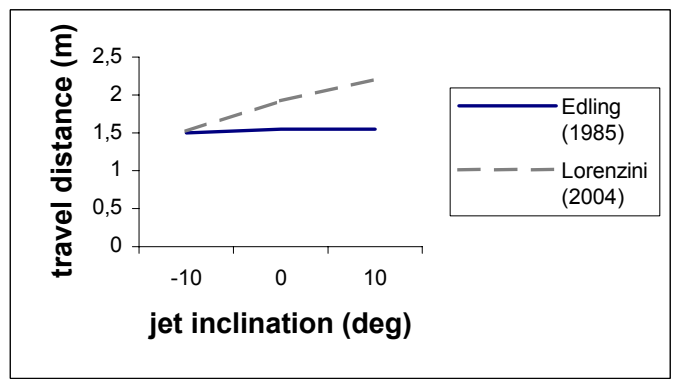

Figure 2: Travel distance of sprinkler droplets: Edling's [5] data compared to Lorenzini's [7]: flow rate $=1.4 \cdot 10^{-4} \mathrm{~m}^{3} \cdot \mathrm{s}^{-1}$; nozzle diameter $3.96 \cdot 10^{-3} \mathrm{~m}$; air temperature $29.4^{\circ} \mathrm{C}$; nozzle height $=2.44 \mathrm{~m}$; droplet diameter $=$ $0.5 \cdot 10^{-3} \mathrm{~m} .\left(\mathrm{R}^{2}=0.912\right)$.

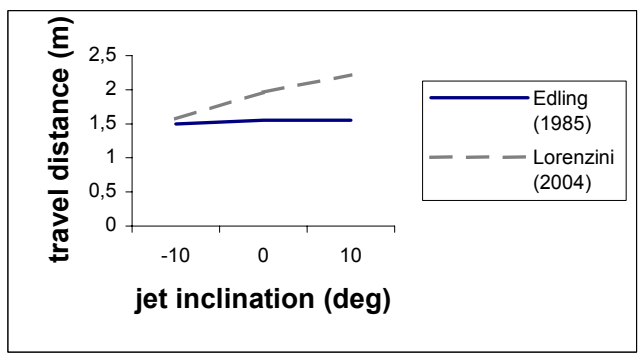

Figure 3: Travel distance of sprinkler droplets: Edling's [5] data compared to Lorenzini's [7]: flow rate $=1.4 \cdot 10^{-4} \mathrm{~m}^{3} \cdot \mathrm{s}^{-1}$; nozzle diameter $3.96 \cdot 10^{-3} \mathrm{~m}$; air temperature $29.4^{\circ} \mathrm{C}$; nozzle height $=3.66 \mathrm{~m}$; droplet diameter $=$ $0.5 \cdot 10^{-3} \mathrm{~m} .\left(\mathrm{R}^{2}=0.918\right)$. 
196 Sustainable Irrigation Management, Technologies and Policies

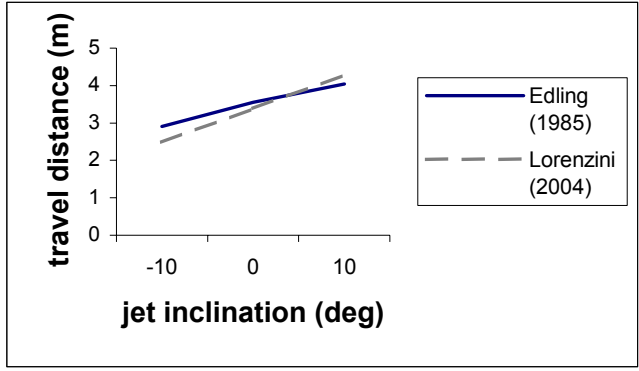

Figure 4: Travel distance of sprinkler droplets: Edling's [5] data compared to Lorenzini's [7]: flow rate $=1.4 \cdot 10^{-4} \mathrm{~m}^{3} \cdot \mathrm{s}^{-1}$; nozzle diameter $3.96 \cdot 10^{-3} \mathrm{~m}$; air temperature $29.4^{\circ} \mathrm{C}$; nozzle height $=1.22 \mathrm{~m}$; droplet diameter $=$ $1.5 \cdot 10^{-3} \mathrm{~m} .\left(\mathrm{R}^{2}=0.997\right)$.

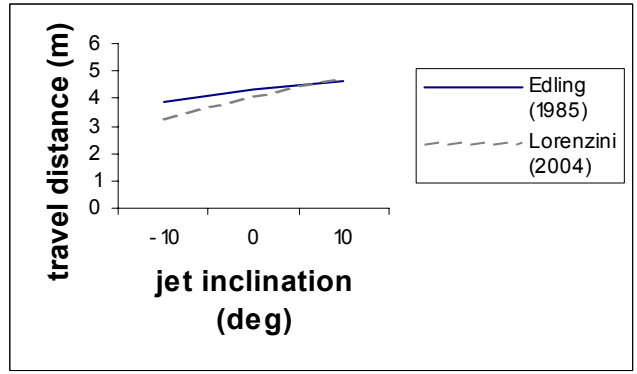

Figure 5: Travel distance of sprinkler droplets: Edling's [5] data compared to Lorenzini's [7]: flow rate $=1.4 \cdot 10^{-4} \mathrm{~m}^{3} \cdot \mathrm{s}^{-1}$; nozzle diameter $3.96 \cdot 10^{-3} \mathrm{~m}$; air temperature $29.4^{\circ} \mathrm{C}$; nozzle height $=2.44 \mathrm{~m}$; droplet diameter $=$ $1.5 \cdot 10^{-3} \mathrm{~m} .\left(\mathrm{R}^{2}=0.997\right)$.

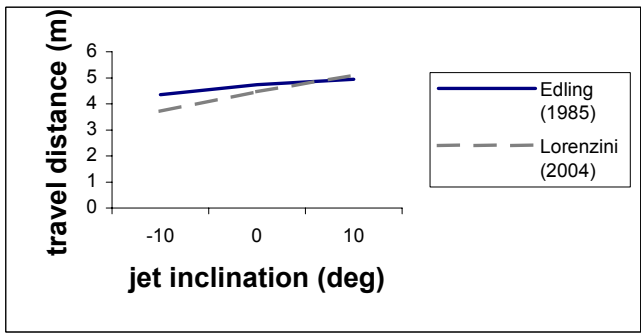

Figure 6: Travel distance of sprinkler droplets: Edling's [5] data compared to Lorenzini's [7]: flow rate $=1.4 \cdot 10^{-4} \mathrm{~m}^{3} \cdot \mathrm{s}^{-1}$; nozzle diameter $3.96 \cdot 10^{-3} \mathrm{~m}$; air temperature $29.4^{\circ} \mathrm{C}$; nozzle height $=3.66 \mathrm{~m}$; droplet diameter $=$ $1.5 \cdot 10^{-3} \mathrm{~m} .\left(\mathrm{R}^{2}=0.995\right)$. 


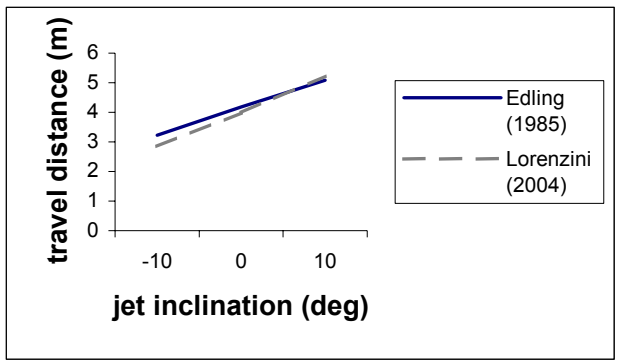

Figure 7: Travel distance of sprinkler droplets: Edling's [5] data compared to Lorenzini's [7]: flow rate $=1.4 \cdot 10^{-4} \mathrm{~m}^{3} \cdot \mathrm{s}^{-1}$; nozzle diameter $3.96 \cdot 10^{-3} \mathrm{~m}$; air temperature $29.4^{\circ} \mathrm{C}$; nozzle height $=1.22 \mathrm{~m}$; droplet diameter $=$ $2.5 \cdot 10^{-3} \mathrm{~m} .\left(\mathrm{R}^{2}=0.999\right)$.

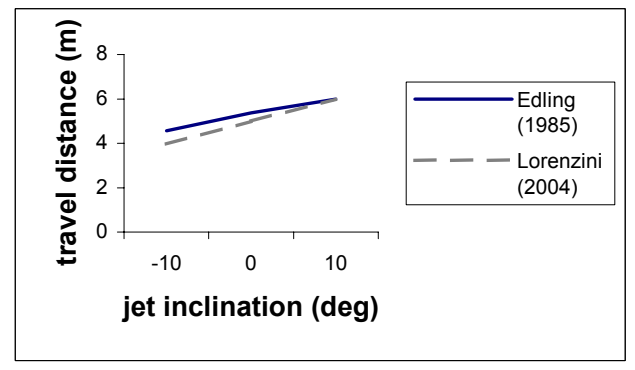

Figure 8: Travel distance of sprinkler droplets: Edling's [5] data compared to Lorenzini's [7]: flow rate $=1.4 \cdot 10^{-4} \mathrm{~m}^{3} \cdot \mathrm{s}^{-1}$; nozzle diameter $3.96 \cdot 10^{-3} \mathrm{~m}$; air temperature $29.4^{\circ} \mathrm{C}$; nozzle height $=2.44 \mathrm{~m}$; droplet diameter $=$ $2.5 \cdot 10^{-3} \mathrm{~m} .\left(\mathrm{R}^{2}=0.998\right)$.

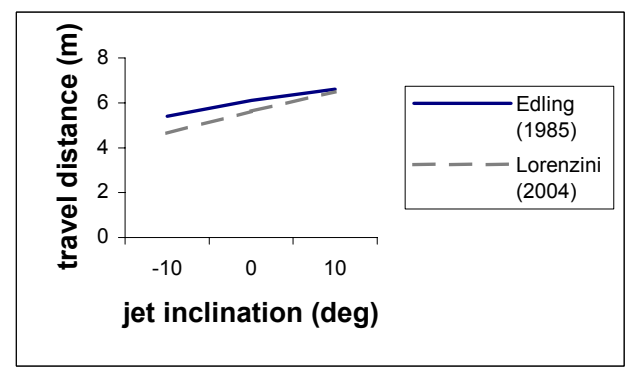

Figure 9: Travel distance of sprinkler droplets: Edling's [5] data compared to Lorenzini's [7]: flow rate $=1.4 \cdot 10^{-4} \mathrm{~m}^{3} \cdot \mathrm{s}^{-1}$; nozzle diameter $3.96 \cdot 10^{-3} \mathrm{~m}$; air temperature $29.4^{\circ} \mathrm{C}$; nozzle height $=3.66 \mathrm{~m}$; droplet diameter $=$ $2.5 \cdot 10^{-3} \mathrm{~m} .\left(\mathrm{R}^{2}=0.998\right)$ 


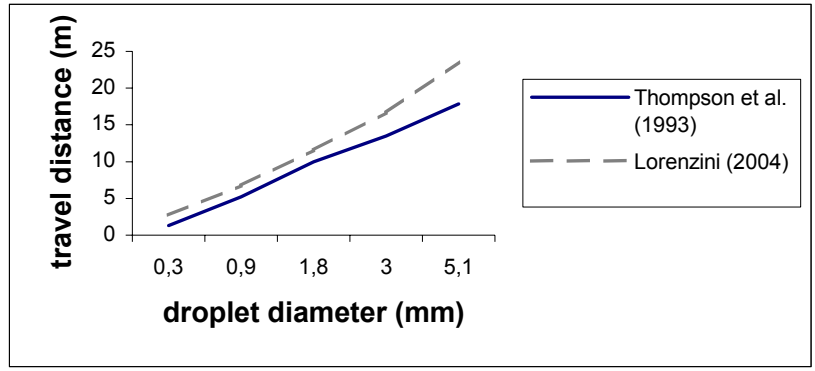

Figure 10: Travel distance of sprinkler droplets: Thompson et al.'s [3] data compared to Lorenzini's [7]: flow rate $=5.5 \cdot 10^{-4} \mathrm{~m}^{3} \cdot \mathrm{s}^{-1}$; nozzle diameter $=4.76 \cdot 10^{-3} \mathrm{~m}$; air temperature $=38^{\circ} \mathrm{C}$; jet inclination $=$ $25^{\circ}$; nozzle height $=4.5 \mathrm{~m} .\left(\mathrm{R}^{2}=0.994\right)$.

Table 1: $\quad$ Time of flight of sprinkler droplets: Thompson et al.'s [3] data compared to that of Lorenzini [7]: flow rate $=5.5 \cdot 10^{-4} \mathrm{~m}^{3} \cdot \mathrm{s}^{-1}$; nozzle diameter $=4.76 \cdot 10^{-3} \mathrm{~m}$; air temperature $=38^{\circ} \mathrm{C}$; jet inclination $=$ $25^{\circ}$; nozzle height $=4.5 \mathrm{~m}$.

\begin{tabular}{|c|c|c|c|c|c|c|}
\hline & \multicolumn{5}{|c|}{ Droplet diameter (mm) } \\
\hline & & 0.3 & 0.9 & 1.8 & 3.0 & 5.1 \\
\hline \multirow{2}{*}{ Time of flight (s) } & $\begin{array}{c}\text { Thompson et al. } \\
\text { (1993) }\end{array}$ & 2.63 & 1.54 & 1.63 & 1.75 & 1.84 \\
\hline & $\begin{array}{c}\text { Lorenzini } \\
\text { (2004) }\end{array}$ & 0.84 & 1.35 & 1.73 & 2.00 & 2.26 \\
\hline
\end{tabular}

\subsection{Computed and experimental droplet evaporation}

A new approach is suggested here for modelling spray evaporation in sprinkler irrigation. Once again, it is based on the analytical model in Lorenzini [7]. In accordance with this model, this section focuses on the effect that air friction has on the aerial evaporation of the droplet and excludes all other contributions, due to the many other parameters, which could have even a strong influence on the process, such as air humidity. This preliminary consideration shows the limit of this particular as a general description of the phenomenon, but highlights the role played by air friction, which has so far not been considered in literature [9]. A few more conditions are added to compute spray evaporation: evaporation is obtained by the total work of the resultant force that is converted into thermal energy; total droplet evaporation occurs at the end of the flight of the droplet and is schematically displayed as a material point. These assumptions determine a restriction to the validity of the results achieved in this section: the final kinetic energy of the droplet is calculated by its initial mass thus allowing an overestimation of the evaporative process. The results obtained are "upper limits" of the real process, aimed at showing the relevance of air friction in spray evaporation for sprinkler irrigation. The model [7, 9] was used again on the data of Edling [5] and Thompson et al. [3]. In contrast to the approach adopted in our research, these works consider a range of parameters. Therefore, qualitative 
comparisons only can be made. Edling's [5] experiment was conducted in many different combinations of conditions, including: flow rate of $1.4 \times 10^{-4} \mathrm{~m}^{3} \cdot \mathrm{s}^{-1}$, nozzle diameter of $7.14 \times 10^{-3} \mathrm{~m}$, jet inclination of $0^{\circ}$, nozzle height of $3.66 \mathrm{~m}$, air temperature of $21.11^{\circ} \mathrm{C}$, relative humidity equal to $20 \%$ and no wind. Those of Thompson et al. [3] were: flow rate of $5.5 \times 10^{-4} \mathrm{~m}^{3} \cdot \mathrm{s}^{-1}$, nozzle diameter of $4.76 \times 10^{-3} \mathrm{~m}$, jet inclination of $25^{\circ}$, nozzle height of $4.5 \mathrm{~m}$, air temperature of $38^{\circ} \mathrm{C}$, relative humidity equal to $20 \%$ and no wind. The comparative results are quoted in Figs. 11 and 12 for a few small-diameter cases.

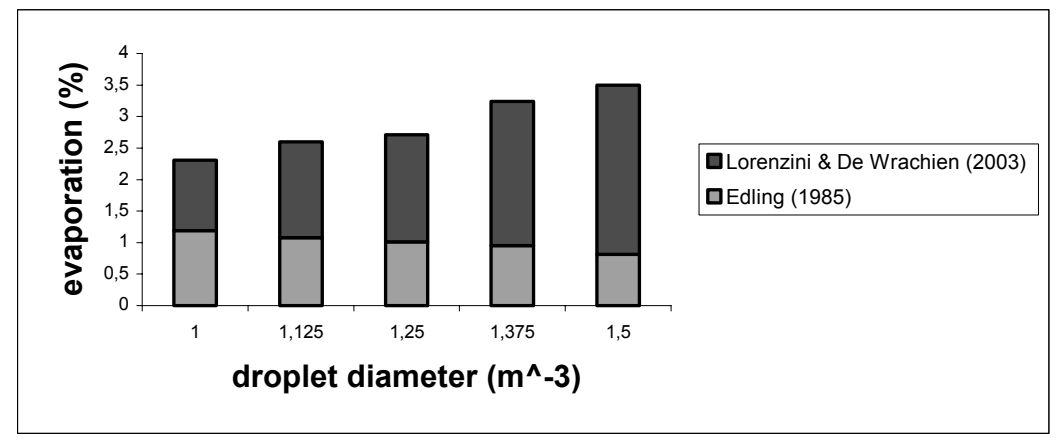

Figure 11: Spray evaporation results: Edling's results [5] compared to those of Lorenzini and De Wrachien [9].

The trends are different because of the over-estimation of the effect of air friction. However, the results obtained by the model and presented are qualitatively correct as they do not show the whole evaporative phenomenon but merely that part of it caused by air friction. In fact, as the friction force depends on the cross sectional area of the droplet, it is reasonable to say that larger droplets undergo larger frictional effects, even if they are not described, apart from with regard to their trends (which are correct), by the data of Figs. 11 and 12 , which are to be considered upper limits, as previously mentioned. It is therefore possible to say that a new window has now been opened in this field, too as until now, air friction may have been unsuitably neglected. From an experimental point of view, of the main research performed in recent decades concerning experimental tests on sprinkler droplet evaporation, the research results published by Zanon and Testezlaf [10], Zanon et al. [11], Molle and Le Gat [12,13], Solomon [14], Tarjuelo et al. [1] were considered in a recent paper by Lorenzini [15]. Less recently, Frost and Schwalen [16] developed a nomograph to estimate evaporation empirically. All these works, characterised by different purposes yet related to the same topic, share the awareness of the difficulty in obtaining a clear result in the process of spray evaporation in sprinkler irrigation, because of the very many parameters mutually affecting one another in obtaining the final result. It is therefore unclear which effect is to be attributed to which parameter. In his paper, Lorenzini [15] proposed an experimental study in which all parameters, except air temperature, were neglected and opportunely set as constant. The problem of minimising experimental error was then faced by a statistical setting of the experimental 
activity itself, obtained by a repetition of each test at least 12 times and by the treatment of the data set by statistic means. Moreover, instead of the usual catch can collection performed by other researchers, it was decided to use a fully circular path of the sprinkler to avoid asymmetric losses in the inversion movements of the device [15]. The results, obtained in a reduced temperature interval varying between 21.0 and $27^{\circ} \mathrm{C}$, for a constant relative air humidity of $94 \%$ and for a water temperature of $15.0^{\circ} \mathrm{C}$, showed an evaporation rate of between 4.15 and $7.73 \%$.

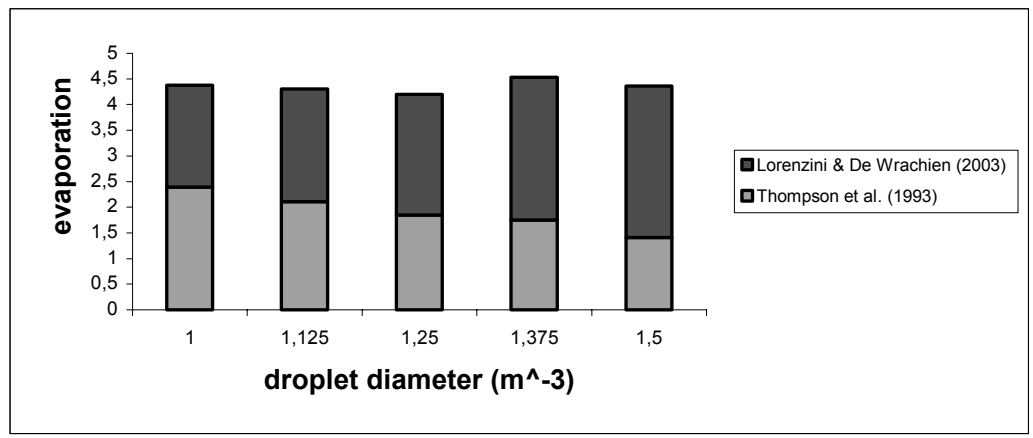

Figure 12: Spray evaporation results: Thompson et al.'s [3] results compared to those of Lorenzini and De Wrachien [9].

Each irrigation test was performed with sprinklers working in steady-state for a time interval of $360 \mathrm{~s}$ and the flow rate delivered by the sprinkler was always equal to $3.025 \times 10^{-4} \mathrm{~m}^{3} \cdot \mathrm{s}^{-1}$. These results are significantly higher than those in Thompson et al. [17], but it should be noted that the climatic conditions of the experimental tests in Lorenzini [15] are far more homogeneous and hence more suitable for singling out each parametrical contribution than those considered in the abovementioned paper. In fact, in Thompson et al. [17], the evaporation measurements, each of which was conducted for a whole day, were obviously affected by usual daily thermal rushes and therefore difficult to interpret. It has to be highlighted that aerial evaporation of irrigation water in sprinkler systems has been very rarely tackled in literature, as most researchers prefer to focus on other phenomena, that are more easily determinable and to which they also attribute the effect actually due to aerial droplet evaporation. This experimental activity, which introduces the novelty of a single-parametric analysis of this problem, proved that the effect of air temperature on sprinkler spray evaporation, previously neglected by most researchers who considered it a less important parameter, in actual fact has an importance that further studies will have to prove in a wider range of air temperature and climatic, cinematic and geometric conditions.

\section{Conclusions}

The present work shows, both theoretically and experimentally, that the thermal fluid dynamics characterisation of water droplets in sprinkler irrigation is a complicated topic, due primarily to the many mutual interactions linking the 
parameters to one another. This difficulty implied, in the approaches available in literature, that the problem could not be fully solved, as excessively complicated systems of equations would be required to obtain a close solution (theoretically) and as the experimental error could not be entirely avoided (experimentally). However, the introduction of some new hypotheses, which are proposed herein, can help in determining a new approach to the problem. This was performed through the:

- introduction of a new and simple analytical method to study the kinematics of the process;

- use of the method to hypothesise a new way of computing sprinkler spray evaporation;

- definition and realisation of a single-parametric experimental test, applied in this case to determine the effect of air temperature on aerial droplet evaporation, to verify whether this direction could be followed to determine the relevance of each parameter to the whole phenomenon.

The research presented in this paper represents a first step from an applicative standpoint, however, from a descriptive point of view it pinpoints an investigative technique that proves to be efficacious in analysing practical situations related to irrigation. This method also entails important consequences for example, on the fundamental question of water waste in agriculture that, as recalled at the start of the paper, becomes increasingly delicate from an ecological standpoint. This is ultimately the most ambitious threshold overcome by this research: to consider water waste in agriculture, in this case with regard to sprinkler irrigation, through the use of a very simple, general (and therefore not connected to case-dependent empirical formulae) method that is applicable in a practical field by farmers with the choice of just a few fundamental environmental parameters, such as the data exiting the sprinkler, and basic weather conditions. The future must of course, develop the technique by expanding the field of the environmental variables considered, however the descriptive capacity of the approach, which has shown good results thus far, already makes it an interesting tool, that must, however, improve the match of the conditions applied to those technically realistic.

\section{Notation}

$\vec{a}=$ acceleration of the droplet, $\mathrm{m} \cdot \mathrm{s}^{-2}$
$A=$ cross sectional area of the
droplet, $\mathrm{m}^{2}$
$f=$ friction factor according to

Fanning

$\vec{F}=$ total force acting on the

system, $\mathrm{N}$

$g=$ acceleration of gravity, $\mathrm{m} \cdot \mathrm{s}^{-2}$

$h=$ nozzle height from ground

level, $\mathrm{m}$

$k=$ friction parameter, $\mathrm{kg} \cdot \mathrm{m}^{-1}$ $m=$ mass of the droplet, $\mathrm{kg}$

$n=$ actual mass of the droplet, $\mathrm{kg}$

$t=$ time, $\mathrm{s}$

$v_{0 x} v_{0 y},=$ initial velocities, $\mathrm{m} \cdot \mathrm{s}^{-1}$

$v_{0}=$ velocity vector of the droplet

exiting the nozzle, $\mathrm{m} \cdot \mathrm{s}^{-1}$

$\dot{x}, \ddot{x}, \dot{y}, \ddot{y}=$ velocities and accelerations (horizontal and vertical direction), $\mathrm{m} \cdot \mathrm{s}^{-1}, \mathrm{~m} \cdot \mathrm{s}^{-2}$

$\alpha=$ exit trajectory of droplet, ${ }^{\circ}$ 
$\rho=$ air density, $\mathrm{kg} \cdot \mathrm{m}^{-3}$

$0($ subscript $)=$ initial value

$\tau=$ droplet time of flight, $\mathrm{s}$

\section{References}

[1] Tarjuelo J.M., Ortega J.F., Montero J., De Juan J.A. (2000) Modelling evaporation and drift losses in irrigation with medium size impact sprinklers under semi-arid conditions. Agric. Water Manage. 43(3) 263-284.

[2] Smolik J., Dzumbova L., Schwarz J., Kulmala M. (2001) Evaporation of ventilated water droplet: connection between head and mass transfer. $J$. Aerosol Sci. 32 739-748.

[3] Thompson A. L., Gilley J.R., Norman J.M. (1993) A sprinkler water droplet evaporation and plant canopy model: II. Model application. Trans. ASAE 36 (3) $743-750$.

[4] Kinzer G. D., and Gunn R. (1951) The evaporation, temperature and thermal relaxation-time of freely falling water drops. J. Meteorol. 8(2) 7183.

[5] Edling R. J. (1985) Kinetic energy, evaporation and wind drift of droplets from low pressure irrigation nozzles. Trans. ASAE 28 (5) 1543-1550.

[6] Kincaid D. C. and Longley T. S. (1989) A water droplet evaporation and temperature model. Trans. ASAE 32 (2) 457-463.

[7] Lorenzini G. (2004) Simplified Modelling of Sprinkler Droplet Dynamics. Biosyst. Eng. 87 (1) 1-11.

[8] Bird R. B., Stewart W. E. and Lightfoot E. N. (1960), Transport phenomena [Fenomeni di trasporto], 188-193, Publisher: Casa Editrice Ambrosiana, Milano, Italy.

[9] Lorenzini G. and De Wrachien D. (2003) phenomenological analysis of sprinkling spray evaporation: the air friction effect. Rivista di Ingegneria Agraria 4 49-54.

[10] Zanon E. R. and Testezlaf R. (1995) Use of load cells in radius layout sprinkler uniformity tests. Proc. XXIV Brazilian Congress of Agric. Eng. pp. 254-263 (M.G. Brazilian Society of Agricultural Engineering).

[11] Zanon E. R. and Testezlaf R. Matsura E. J. (2000) A data acquisition system for sprinkler uniformity testing. Appl. Eng. Agric. 16 (2) 123-127.

[12] Molle B. and Le Gat Y. (2000a) Model of Water Application under Pivot Sprinkler. I: Theoretical Grounds. J. Irrig Drain Eng. 126(6) 343-347.

[13] Molle B. and Le Gat Y. (2000b) Model of Water Application under Pivot Sprinkler. II: Calibration and Results. J. Irrig Drain Eng. 126(6) 348-354.

[14] Solomon K. (1979) Variability of sprinkler coefficient of uniformity test results. Trans. ASAE 22(5) 1070-1080.

[15] Lorenzini G. (2002) Air Temperature effect on spray evaporation in sprinkler irrigation. Irrig. Drain. 51 (4) 301-309.

[16] Frost K. R., Schwalen H.C. (1955) Sprinkler evaporation losses. Agric. Eng. 36 (8) 526-528.

[17] Thompson A. L., Martin D. L., Norman J. M., Tolk J. A., Howell T. A., Gilley J. R., Schneider A. D. (1997) Testing of water loss distribution model for moving sprinkler systems. Trans. ASAE $\mathbf{4 0}$ (1) 81-88. 